Research Article

\title{
Prediction of new chromene-based inhibitors of tubulin using structure-based virtual screening and molecular dynamics simulation methods
}

\author{
Hassan Aryapour $^{\mathrm{a}, *}$, Maryam Dehdab ${ }^{\mathrm{b}}$, Farzin Sohraby ${ }^{\mathrm{a}}$, Afshar Bargahi $^{\mathrm{c}}$ \\ a Department of Biology, Faculty of Science, Golestan University, Gorgan, Iran \\ ${ }^{\mathrm{b}}$ Young Researchers and Elite Club, Bushehr Branch, Islamic Azad University, Bushehr, Iran \\ ${ }^{\mathrm{c}}$ Persian Gulf Marine Biotechnology Research Center, Bushehr University of Medical Sciences, Bushehr, Iran
}

\section{A R T I C L E I N F O}

\section{Article history:}

Received 18 June 2017

Received in revised form 4 September 2017

Accepted 21 September 2017

Available online 27 September 2017

\section{Keywords:}

Chromene

Microtubule

Tubulin

Cancer

Docking

Molecular dynamics simulation

Virtual screening

\begin{abstract}
A B S T R A C T
Multidrug resistance (MDR) is one of the serious problems in cancer research that causes failure in chemotherapy. Chromene-based compounds have been proven to be the novel anti-MDR agents for inhibiting proliferation of tumor cells through tubulin polymerization inhibition of by binding at the colchicine binding site. In this study, we screened a chromene-based database of small molecules using physicochemical, ADMET properties and molecular docking to identify potential hit compounds. In order to validate our hit compounds, molecular dynamics simulations and related analysis were carried out and the results suggest that our hit compounds (PubChem CIDs: 16814409, 17594471, 57367244 and 69899719) can prove to be potential inhibitors of tubulin. The in silico results show that the present hits, like colchicine, effectively suppressed the dynamic instability of microtubules and induced microtubuledepolymerization and cell cycle arrest.
\end{abstract}

(c) 2017 Elsevier Ltd. All rights reserved.

\section{Introduction}

Cancer is the result of uncontrolled cell proliferation, where transformed normal cells (malignant cells) aggressively divide and spread to other areas of human body through metastasis. During the last decades, no significant improvements have been made in the field of approved conventional therapeutic drugs due to their side effects. Limited clinical effectiveness, probable resistance and toxicity remain serious problems, associated with the existent anticancer drugs (Temraz et al., 2014). The World Health Organization (WHO) had estimated 14.1 million cancer cases and up to 8.2 million cancer-related mortality cases in 2012 (Bray et al., 2013).

The emergence of drug-resistant tumor cells is rapidly becoming a major worldwide problem. Therefore, finding new drugs to overcome this resistance has become one of the most important goals of recent medical researches. In general, the cancer cells exhibit unusual inhibition of apoptosis, hence many commonly used chemotherapeutic drugs induce tumor cell

\footnotetext{
* Corresponding author.

E-mail address: h.aryapour@gu.ac.ir (H. Aryapour).
}

apoptosis (Waxman and Schwartz, 2003; Patil et al., 2013). The pro-apoptotic chemotherapeutic agents that interfere directly with the tubulin system, are the most successful and commonly prescribed anticancer therapeutics. The importance of tubulin protein in mitosis and cell division, makes it an important target of anticancer drugs. These drugs can react or bind at various sites on tubulin; such as their sulfhydryl (-SH) groups. It is evident that many new anticancer chemotherapeutic drugs function by targeting $\alpha / \beta$-tubulin. The colchicine binding site is one of the most important pockets for potential tubulin inhibitors for the development of apoptotic-inducing chemotherapeutic agents. Over the last few years, our Organic Chemistry group has been focused on the development of new eco-friendly methods for the synthesis of bioactive heterocyclic compounds, including the preparation of chalcones, flavonols, and chromene derivatives (Saffari et al., 2014; Aryapour et al., 2012a,b, 2011).

The chromenes inhibit tubulin polymerization and bind at or close to the colchicine binding site. Chromene scaffold is an important class of benzopyran derivatives found in plants, including edible vegetables and fruits (Curini et al., 2006). Chromenes and their derivatives play a pivotal role in the scope of natural and synthetic organic chemistry because of their 
biological and pharmacological properties (Green et al., 1995), in the form of anti-HIV (Smith et al., 1998; Mungra et al., 2011), anticancer, anticoagulant, diuretic, spasmolytic, anti-anaphylactic (Foye and Piccin, 1991), antibacterial and fungal agents (Khafagy et al., 2002). Chromene-based compounds have been proven to be one of the novel anti-MDR agents that inhibit proliferation of tumor cells through the inhibition of tubulin polymerization by binding at the colchicine binding site (Patil et al., 2013).

Two main approaches are currently available for drug discovery research, namely, high throughput screening (HTS) and computeraided drug design. Despite the development in HTS approach, the screening of all compounds, using biological assays is still very expensive, difficult and time-consuming. In the last two decades, advances in computational programming and processing power have made the structure-based virtual screening (SBVS) an important tool to identify starting points for the discovery of new potentially active molecules that can be called "Hit" compounds (Tanrikulu et al., 2013; Muegge, 2008; Walters et al., 1998; M.I.O.S, 2006).

In this study, we employed a set of in silico methods such as docking procedure, virtual screening techniques and molecular dynamics (MD) simulation as well as the analysis of Absorption, Distribution, Metabolism, and Excretion (ADME), for filtering a large set of chromene compounds in order to find new scaffolds for colchicine-binding site of tubulin. Molecular dynamics simulation techniques were used to predict more reliable protein-ligand complex structures. The results described here, can provide a valuable dataset of chromene inhibitors for future experimental studies.

\section{Materials and methods}

\subsection{Protein preparation}

The X-ray structure of $\alpha / \beta$-tubulin (PDB ID: 4O2B, resolution: $2.30 \AA$ (Prota et al., 2014)) was obtained from Protein Data Bank (Berman et al., 2000). First, additional parts of PDB file, except for chains d, c and related heteromolecules were removed. Missing residues and parts of tubulin were added and refined using UCSF Chimera graphical interface in MODELLER software (Fiser et al., 2000). Then, final complex was simulated by GROMACS to reach the equilibrium state

\subsection{Ligand selection and preparation for docking}

Structure-based virtual screening was employed to screen two chemical databases: ZINC (Irwin and Shoichet, 2005) and PubChem (Kim et al., 2016) to discover novel inhibitors of 4O2B. Totally, 117 thousand chromene-based structures were downloaded from ZINC and PubChem databases. Because of the existence of non-chromene compounds in downloaded file, all chromene-based isomers were extracted from these files and the duplicates were excluded.

\subsection{Filtering and virtual screening}

Virtual screening of known chemical databases is a fast and accurate method, which serves the purpose of identifying potential hits, suitable for further developments. Before the virtual screening by docking, compounds that were expected to be unsuitable drugs or those having toxic and poor ADME properties, were firstly eliminated by using a series of simple pharmacodynamic or pharmaco-kinetic descriptors; such as Lipinski's Rule of Five (RO5) (Lipinski et al., 2001). Most of the drugs failed in developmental stages of clinical trials, due to their poor pharmacokinetic parameters. R05states that a molecule is likely to be orally active if it satisfies the following conditions: (a) the molecular weight should be below 500, (b) the calculated octanol/ water partition coefficient $(\log \mathrm{P})$ should be less than 5 , (c) number of hydrogen bond donors (e.g.; $\mathrm{OH}$ and $\mathrm{NH}$ groups) should not exceed 5, and (d) number of hydrogen bond acceptors (notably $\mathrm{N}$ and $\mathrm{O}$ ) should not exceed 10 . There are exceptions to this rule as well: a compound is likely to be orally active as long as not more than one rule is broken. Extensions of RO5 state that polar surface area should likely be less than or equal to $140 \AA^{2}$ and the number of rotatable bonds should be less than or equal to 10 (Veber et al., 2002). Molecular polar surface area (PSA) is an important property in calculating the transport properties of drugs. Surface areas of polar atoms in a molecule, usually oxygen, nitrogen and attached hydrogens are summed up to calculate the polar surface area as a sum of fragment contributions as per the previously reported method (Ertl et al., 2000). In the present study, the RO5, ADME and toxicological (collectively ADMET) properties for compounds were performed using DruLiTo software, and CypRules and admetSAR servers (Cheng et al., 2012; Shao et al., 2015). Compounds that passed the RO5 were further assessed for the prediction of ADMET properties. ADMET descriptors, including aqueous solubility, blood-brain barrier (BBB) penetration, cytochrome P450, hepatotoxicity, human intestinal absorption (HIA), and plasma-protein binding (PPB) were estimated for RO5 testified. Finally, the total number of compounds reached 2204 after filtering. The final structures were prepared for molecular docking by merging nonpolar hydrogens, assigning gasteiger charges on each atom. AutoDock Vina software, which is one of the most accurate docking techniques, was used for docking computations (Trott and Olson, 2010).

\subsection{Molecular docking setup}

Docking process is one of the most widespread virtual screening approaches in computational drug discovery research. The docking technique consists of sampling the ligand orientations within targets' binding site to form a stable complex. The hit compounds, obtained from the virtual screening analysis, were used in the docking calculation to investigate the detailed intermolecular interactions between a ligand and its receptor. The molecular docking studies were carried out to explore the interaction mechanisms between virtual hits and the receptor.

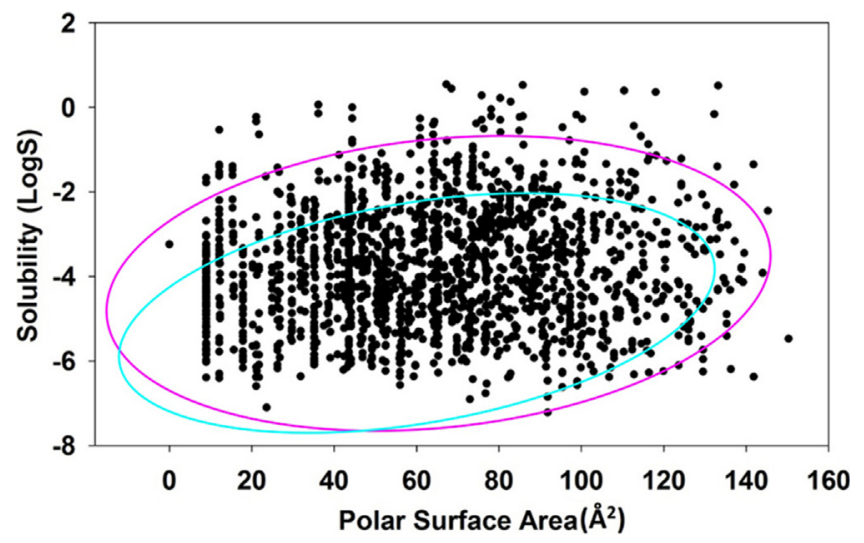

Fig. 1. Plot of polar surface area (PSA) against solubility (LogS) after the filtration of compounds, based on physicochemical properties. The area encompassed by the ellipse is a prediction of good absorption by blood-brain barrier (Cyan color) and Caco-2 Permeability (Pink color) with no violation of ADMET properties.(For interpretation of the references to colour in this figure legend, the reader is referred to the web version of this article.) 
Table 1

Results of molecular docking scores and X-score of top 4 hit compounds inside the binding site of colchicine.

\begin{tabular}{lll}
\hline Hits & Binding affinity $\left(\mathrm{Kcal} \mathrm{mol}^{-1}\right)$ & X-score \\
\hline 17594471 & -10.7 & 6.78 \\
69899719 & -10.3 & 6.36 \\
16814409 & -9.6 & 6.24 \\
57367244 & -10.2 & 6 \\
Colchicine & -9.3 & 5.25 \\
\hline
\end{tabular}

The validation of docking protocol is essential to avoid the false positive results of molecular docking. This was done by the calculation of Root Mean Square Deviation (RMSD) value with respect to the co-crystallized ligands. If the RMSD value is equal to or less than $2.0 \AA$ between the real and the best-scored conformations he docking process will be considered successful (Wang et al., 2003). In order to validate the docking procedure, native ligand of the crystal structure was removed from binding site and was re-docked into the binding site of 402B. The RMSD between the predicted and observed conformations of native ligand of crystal structure (PDB ID: 4O2B) is equal to $0.6 \AA$. This value that suggests that the reliability of the AutoDock Vina docking mode in reproducing the experimentally observed binding mode for 4O2B inhibitors and the parameter, set for docking, is reasonable to reproduce the $\mathrm{X}$-ray structure. On the basis of this method, we can conclude that the docking method was validated and reliable for further calculations.

Default parameters in AutoDock Vina that were followed for the docking studies of virtual hits included the processing of protein and ligand. All the virtual hits were docked into the active site of tubulin, (4O2B) using AutoDock Vina program. During protein preparation, ligand and solvent molecules were deleted, and the hydrogen atoms were added to it. Each protein structure and ligand was converted from a PDB file type to pdbqt format and a grid box with the specified coordinates $(X=115.373, Y=90.489$ and $\mathrm{Z}=46.358$ ) was generated at the centroid of the active site.
For each docked ligand, scoring function values were obtained at ten different binding confirmations. Each pose suggests the best binding conformation, energy and binding site of the drug into tubulin (4O2B) in a cycle of runs. Finally, the lowest binding energy pose was selected among 10 docking poses for downstream analysis. Following in silico molecular docking, the receptor-ligand complexes were analyzed using PoseView server (Stierand and Rarey, 2007) to delineate the amino acid residues, which are involved in receptor-ligand interaction.

For comparison and validation of docking results, we used a consensus scoring function of X-Score (Wang et al., 2002). This program has three built-in scoring functions, HPScore, HMScore, HSScore and calculates the negative logarithm for the dissociation constant of the ligand-protein as the average of three scoring functions, and predicts the binding affinity (Kcal/mol) of the ligand. All the default parameters of X-Score program were used during this analysis.

\subsection{Molecular dynamics simulation}

MD simulations are useful for filling-in the details where experimental methods cannot (Durrant and McCammon, 2011). MD techniques have been applied to predict more reliable proteinligand complexes. MD simulations can treat both ligand and protein in a flexible way, allowing for an induced fit of the receptorbinding site around the newly introduced ligand. In addition, the effect of explicit water molecules can be studied directly, and very accurate binding free energies can be obtained via this method. In the present study, in order to further validate the rationality of the molecular docking results and the structural stability of the 4O2Bhits complexes, MD simulation was performed with GROMACS 5.0.5 software package (Van Der Spoel et al., 2005). The topology files of the 4O2B were directly generated by GROMACS, whereas SwissParam server was used to generate topology files of the hit compounds (Zoete et al., 2011).

The general charmm27 force field (Brooks et al., 2009) was used for the hits and the protein. For the MD simulation studies, all of the complexes were immersed in the center of a triclinic box with a
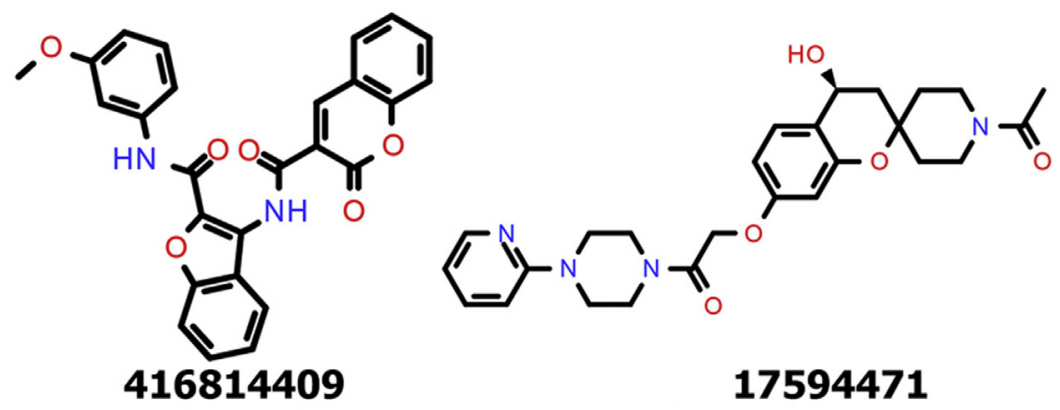

416814409

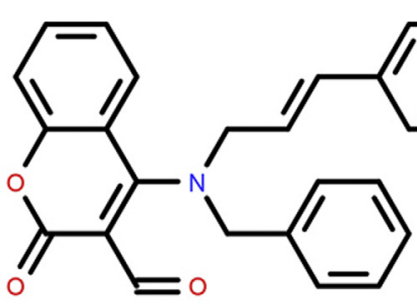

$\mathbf{5 7 3 6 7 2 4 4}$
17594471

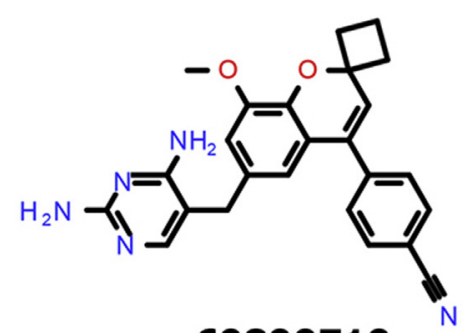

69899719

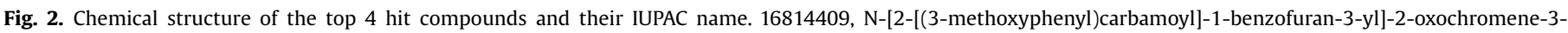

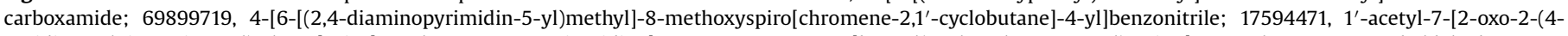
pyridin-2-ylpiperazin-1-yl)ethoxy]spiro[3H-chromene-2,4'-piperidine]-4-one; 57367244, 4-[benzyl(3-phenylprop-2-enyl)amino]-2-oxochromene-3-carbaldehyde. 


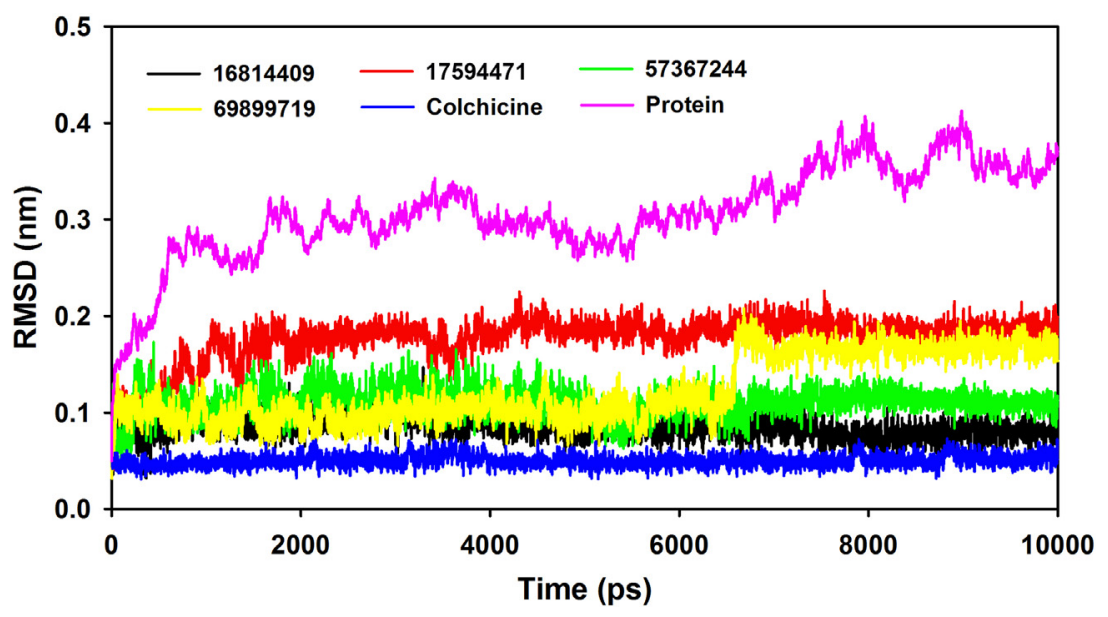

Fig. 3. The RMSD of $402 \mathrm{~B}$ complexes during 10 ns MD simulations.

margin of $1 \mathrm{~nm}$ and the systems were filled with water using TIP3P water model. The box dimensions were $87.389 \AA \times 114.695 \AA$ $\times 126.027 \AA$ with 303 SPC water molecules. Enough sodium and chloride ions were added to neutralize the charges in the systems. Each complex was firstly energy-minimized, using steepest descent minimization algorithm, until the maximum force was found to be smaller than $1000 \mathrm{~kJ} / \mathrm{mol} \mathrm{nm}^{-1}$. All the covalent bonds were constrained using the LINCS (Linear Constraint Solver) algorithm (Hess et al., 1997) to maintain constant bond lengths. The electrostatic interactions were treated using the Particle Mesh Ewald (PME) method (Essmann et al., 1995). The cut-off radii for coulomb and van der Waals interactions were set to $1.2 \mathrm{~nm}$. The modified Berendsen (velocity rescaling) thermostat and Parrinello-Rahman barostat were applied for $100 \mathrm{ps}$ to keep the system in stable environmental conditions ( $310 \mathrm{~K}, 1 \mathrm{Bar})$. Finally, after equilibration of the systems, $10 \mathrm{~ns}$ MD were carried out under the periodic boundary conditions, set at XYZ coordinates to ensure that the atoms had stayed inside the simulation triclinic box and the subsequent analyses were then performed.

RMSD, Root Means Square fluctuation (RMSF), radius of gyration ( $\mathrm{Rg}$ ) and the number of $\mathrm{H}$-bonds formed between the ligands and the protein, were also evaluated. In addition, to estimate the binding free energies, the trajectories were used as input file for g_mmpbsa package to estimate the binding free energies (Kumari et al., 2014). The frames were extracted every
$100 \mathrm{ps}$. Binding free energy for each ligand was calculated from the difference in free energies for the complexed and un-complexed reactants, according to the following equation:

$\Delta \mathrm{G}_{\text {bind }}=\mathrm{G}_{\text {complex }}-\mathrm{G}_{\text {ligand }}-\mathrm{G}_{\text {receptor }}$

\section{Results and discussion}

\subsection{Database screening}

A set of 117 thousand compounds were first filtered for druglike properties, using RO5. In the second level of drug-likeness screening, ADMET properties were calculated for all hit compounds. The filtered molecules, based on RO5 and ADMET properties, provided a library of 2204 molecules (Fig. 1).

Then, we used the AutoDock Vina docking program to screen these molecules. Docking analysis of compounds was carried out to identify the hits compounds that have lowest binding affinity with 4O2B (Table 1). The reference ligand of 402B structure exhibits a binding affinity value of $-9.3 \mathrm{Kcal} / \mathrm{mol}$ and its corresponding $\mathrm{X}$ score was 5.25. Among these compounds, we selected inhibitors having a binding affinity value higher than $-9.3 \mathrm{Kcal} / \mathrm{mol}$ and the $\mathrm{X}$-score value of 5.25. A total of 15 hits showed a binding score and $\mathrm{X}$-score, much better than the reference ligand. After a careful analysis based on the non-covalent interactions between the

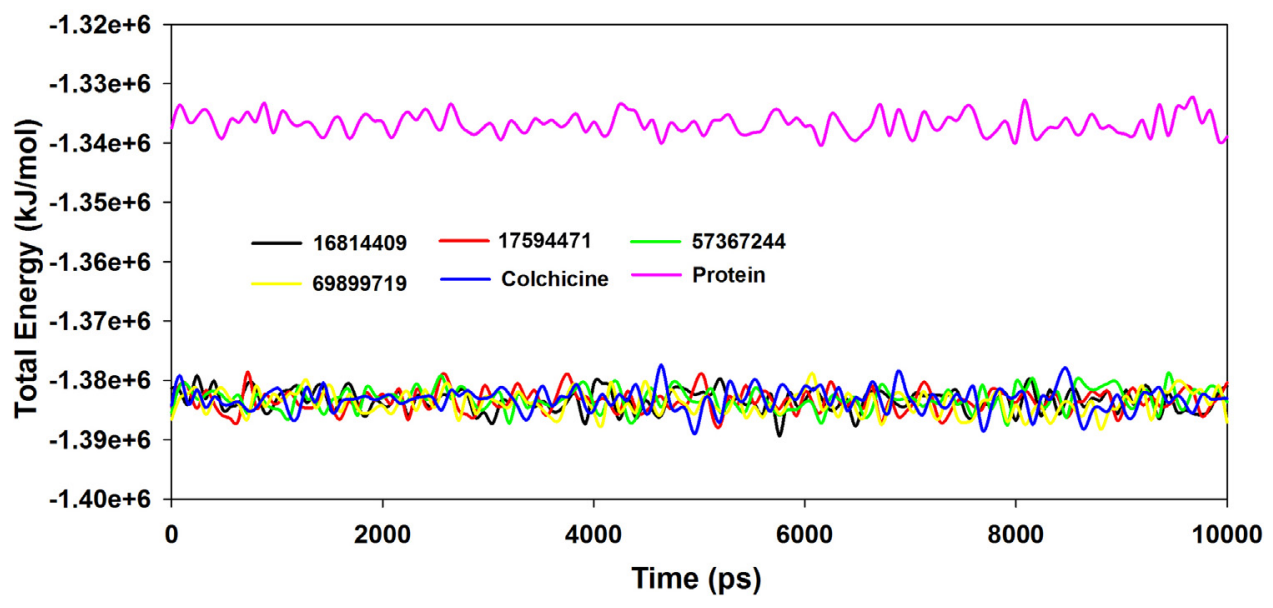

Fig. 4. Total energy plots of $\alpha \beta$-tubulin in complex with the top 4 hit compounds during MD simulations. 

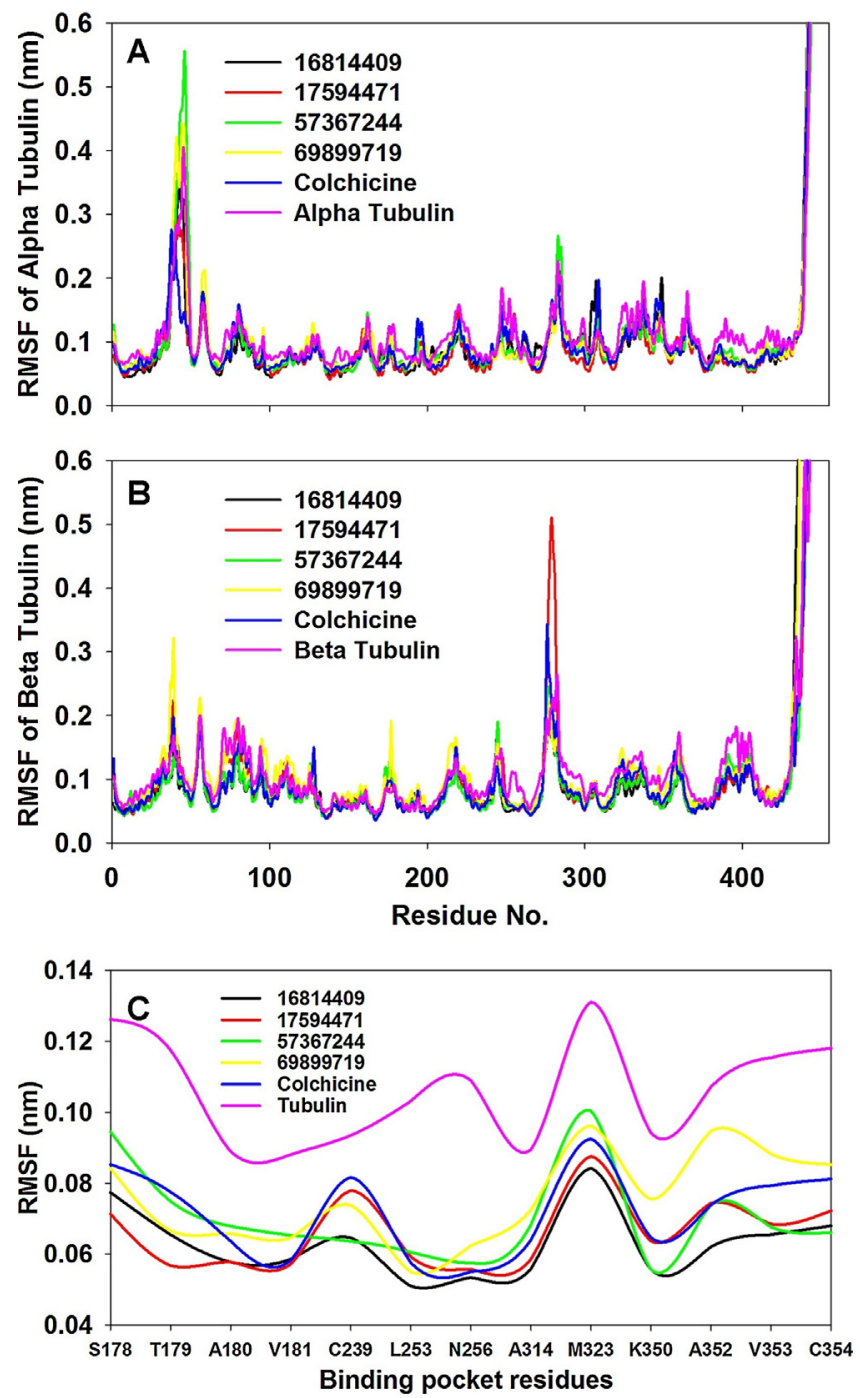

Fig. 5. RMSF of $\mathrm{C} \alpha$ atoms of $\alpha \beta$-tubulin in complex with top 4 hit compounds during $10 \mathrm{~ns}$ MD simulations. A) Alpha subunit; B) Beta subunit and C) Binding site residues.

compounds and the receptor and close structure similarity to colchicine (reference ligand), we selected top 4 scoring hits for the further analyses (Fig. 2).
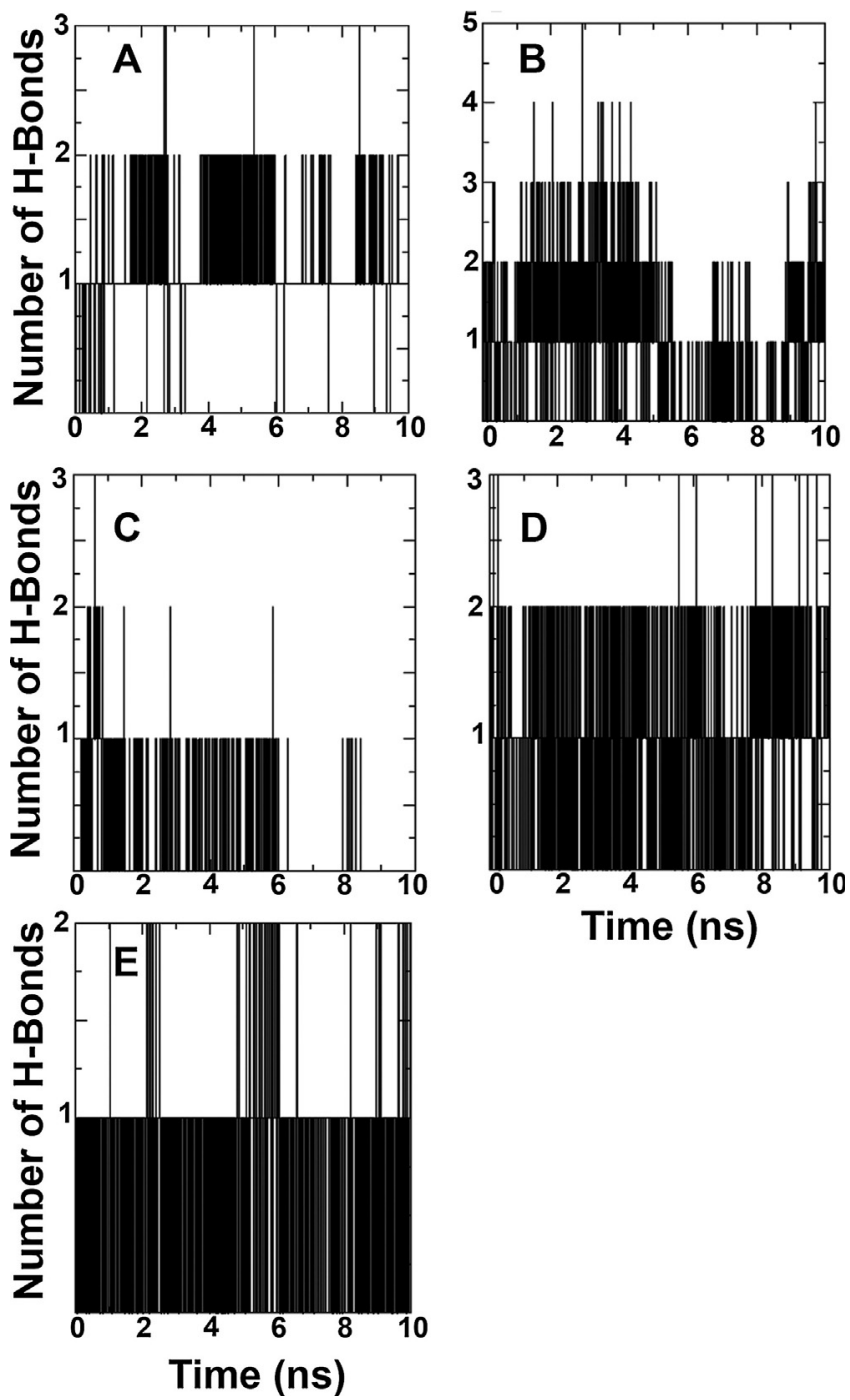

Fig. 7. Hydrogen bond formation in hit-protein complexes as a function of time. A) 16814409, B) 17594471, C) 57367244 , D) 69899719 and E) Colchicine.

\subsection{Molecular dynamics simulation of the top 4 hits}

MD simulations was performed to further confirm the reliability of docking results and to obtain a more precise

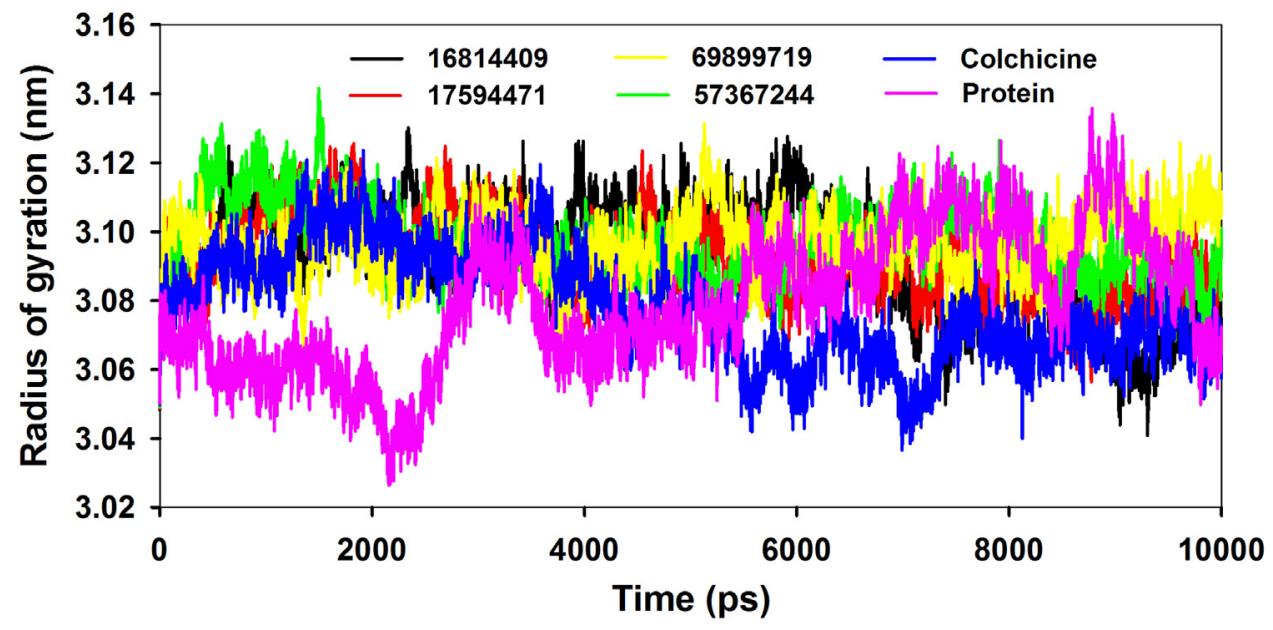

Fig. 6. Time trajectory of radius of gyration of the $\alpha \beta$-tubulin dimer in complex with top 4 hits. 


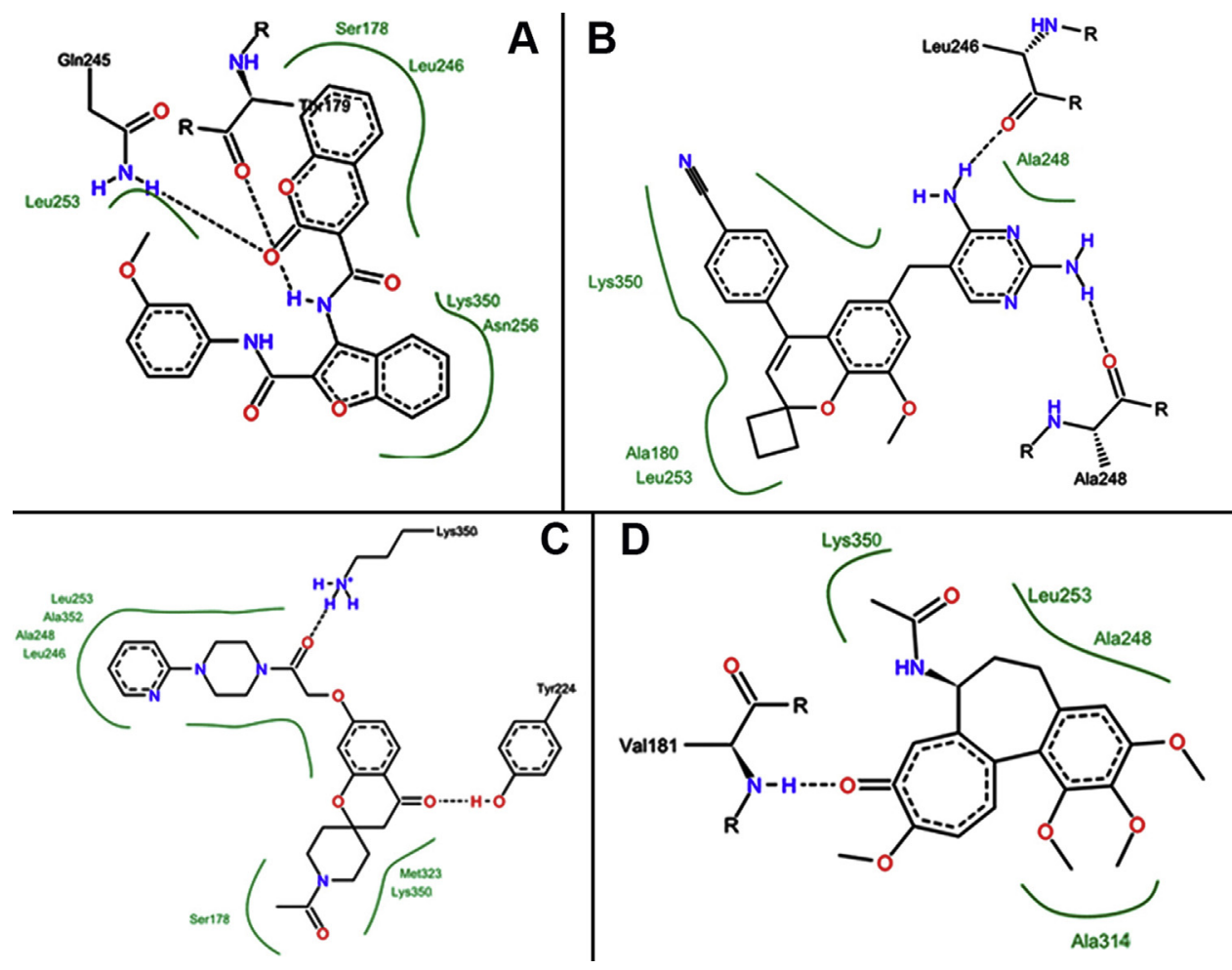

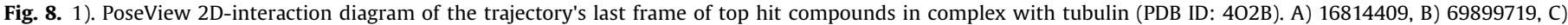

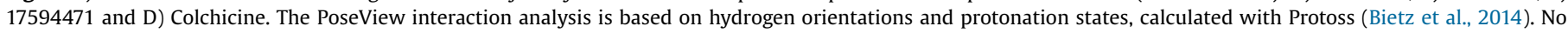

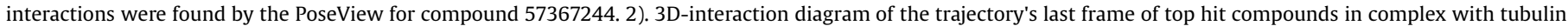
(PDB ID: 4O2B). E) 16814409, F) 69899719, G) 17594471, H) 57367244 and I) Colchicine.

ligand-receptor model in a state close to the natural conditions and to explore key residues at the active site of receptor during the protein-ligand complex simulation. Although, MD simulation is computationally expensive, but it can take the protein flexibility and ligand into consideration. The docked structures of the four selected inhibitors were used as initial structures for MD simulations. After completion of simulation process, the four complexes were checked for equilibration of total energy and RMSD. Analysis of trajectories revealed that all systems in the simulation were well-equilibrated and remained stable throughout the simulation of $10 \mathrm{~ns}$ (Fig. 3). The total energy plot shows that the potential energy for all of hits remained more negative as compared to only protein, which indicates that the complexes were stable during $10 \mathrm{~ns}$ simulation run (Fig. 4). To confirm the stability of prepared protein in solvent system, the RMSD of backbone, was calculated after $10 \mathrm{~ns}$ simulation. The final RMSD value of tubulin (4O2B) remained below $0.15 \mathrm{~nm}$ before and after the simulation analysis, which indicates that the protein structure is more rigid in the presence of inhibitors during simulation. All complexes, except 69899719 , had relatively smooth curves; While for 69899719 , the RMSD value suddenly jumped from 0.1 to $0.2 \dot{A}$ at $6.6 \mathrm{~ns}$, which indicated a slight conformational change in 69899719 complex. These results indicate that the dynamic instability of microtubule is suppressed by Hits. Dynamic instability plays a key role in determining the organization of microtubules into arrays, and these arrays vary throughout the cell cycle and assist the cell division as well.

Also, the RMSF plot of the C $\alpha$ atom of each residue in the Hits402B complexes was calculated to reveal the flexibility of tubulin backbone (Fig. 5). The high RMSF value indicates more flexibility, whereas the low RMSF value shows limited movements during simulation, in relation to its average position over time. The results showed that the RMSF of the Hits-4O2B complexes is significantly lower than that of only the protein, and some of the residues constituting the binding pocket of protein, had lower RMSF values as compared to only tubulin (Fig. 5C). Therefore, the lower RMSF values of the complexes indicates the lesser extent of conformational changes and it suppressed the dynamic instability of microtubules as well.

The Radius of gyration ( $\mathrm{Rg}$ ) measures the distance of a region's parts from its center of gravity. It is defined as the mass weighted root mean square distance of a collection of atoms from their common center of mass or in other words, how packed a certain region is. This allows theoretical scientists to check their models against reality. As it is evident from Fig. 6, the $R g$ of each complex remained stable during simulation run. This reveals the stabilization and non-significant conformational changes in the structure of all complexes except the tubulin that has a dynamic structure.

A potent inhibitor is thought to form the hydrogen bonding interactions with protein. Hydrogen bonds play a vital role in stabilizing the protein-ligand complexes. Higher number of intermolecular hydrogen-bond interactions leads to greater stability of protein-ligand complex. In the present investigation, hydrogen bond analysis was performed to depict the stability of top 4 hits and 4O2B (Fig. 7). In the case of 17594471, hydrogen bond interactions reached a maximum of four and two to three of them remained for most of the time. While, in the case of 69899719, the number of hydrogen bonding interactions reached up to three for some times, except other two. In the case of 16814409 , the number of hydrogen bonding interactions reached three and the two of them remained between 6 and 10 ns. Complex of hit 57367244 and 4O2B showed two hydrogen bonds in the simulation run, in which 

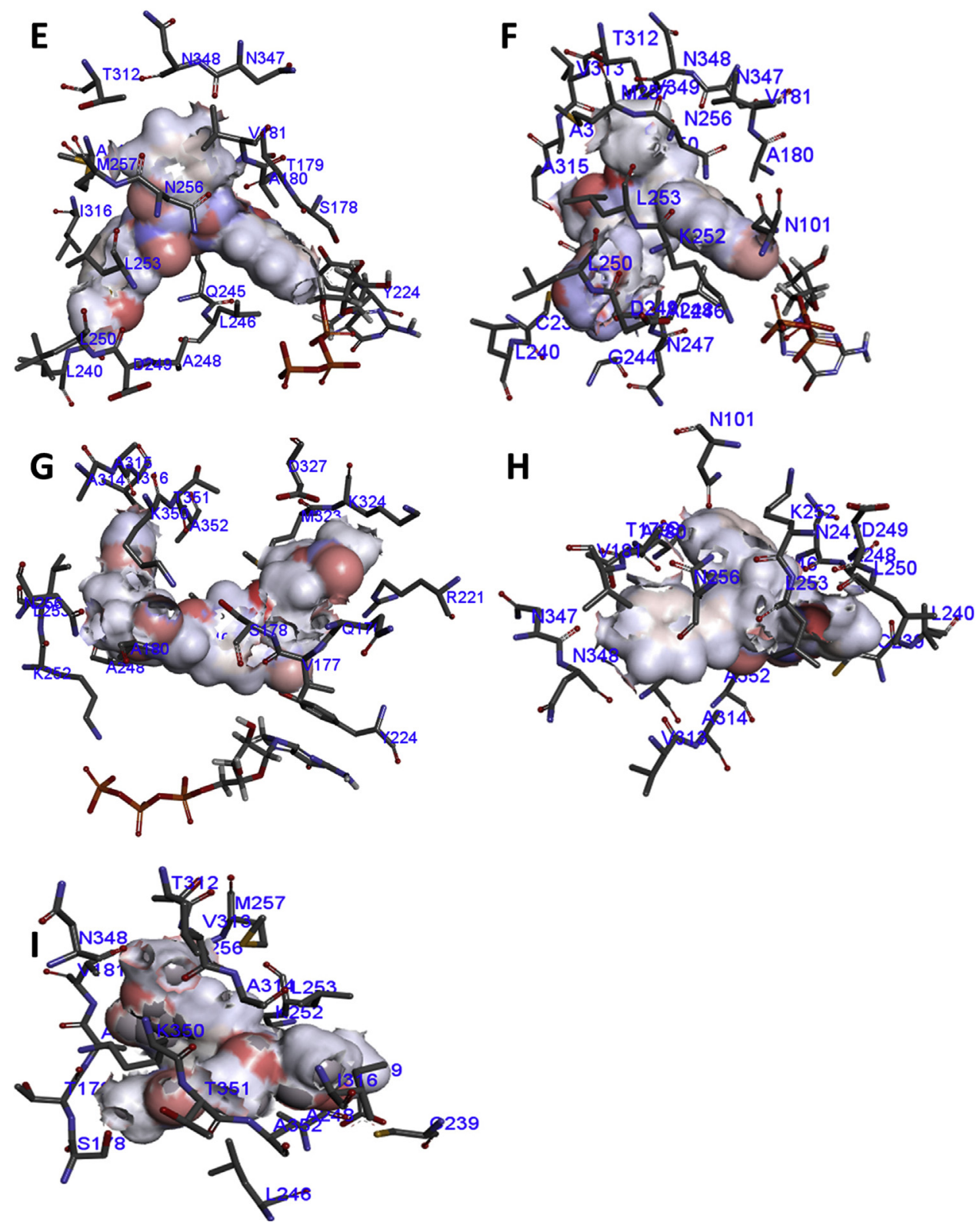

Fig. 8. (Continued)

one or none were maintained between $6 \mathrm{~ns}$ and $10 \mathrm{~ns}$, respectively. Whereas, colchicine-402B complex exhibited one H-bond throughout the simulation time period, which indicates that all of the hits except 57367244 showed stable and strong H-bonds with 402B.

For a better understanding of interactions between hits and the residues of 402B, a plot was drawn using PoseView. As evident from Fig. 8, Val181A residue formed a hydrogen bond with a length of $3.23 \AA$ in Colchicine-402B complex. While Leu246A and Ala248A residues formed hydrogen bonds with lengths of 2.73 and 2.8 Å in 69899719, respectively. Lys350A and Tyr224A residues formed hydrogen bonds with the lengths of 3.06 and $2.71 \AA$ in 17594471, respectively. Finally, Gln245A and Thr179A residues formed hydrogen bonds with a length of 3.07 and $2.91 \AA$ in

Table 2

VDW, Electrostatic, Polar solvation, SASA and binding free energies of the hit compounds in complex with tubulin. All values were calculated as kJ/mol.

\begin{tabular}{|c|c|c|c|c|c|}
\hline Hits & van der Waal energy & Electrostatic energy & $\begin{array}{l}\text { Polar solvation } \\
\text { energy }\end{array}$ & SASA energy & Binding energy \\
\hline 16814409 & $-237.7 \pm 1.4$ & $-70.7 \pm 1.8$ & $214.4 \pm 2.2$ & $-25.5 \pm 0.1$ & $-119.4 \pm 1.5$ \\
\hline 17594471 & $-224.5 \pm 1.3$ & $-16.8 \pm 2.4$ & $208.0 \pm 2.8$ & $-26.1 \pm 0.1$ & $-59.5 \pm 3.1$ \\
\hline 57367244 & $-240.5 \pm 1.1$ & $-56.9 \pm 2.0$ & $188.8 \pm 2.2$ & $-22.5 \pm 0.1$ & $-131.2 \pm 1.6$ \\
\hline 69899719 & $-229.6 \pm 2.3$ & $-954.7 \pm 3.8$ & $236.5 \pm 6.0$ & $-24.7 \pm 0.1$ & $-972.6 \pm 3.8$ \\
\hline Colchicine & $-238.9 \pm 1.4$ & $-60.6 \pm 1.0$ & $161.2 \pm 1.0$ & $-21.9 \pm 0.1$ & $-116.1 \pm 1.9$ \\
\hline
\end{tabular}


Table 3

Prediction of some of ADMET properties of hit compounds using admetSAR server.

\begin{tabular}{|c|c|c|c|c|c|}
\hline Models PubChem CID & $\begin{array}{c}6167 \\
\text { (Colchicine) }\end{array}$ & 69899719 & 57367244 & 17594471 & 16814409 \\
\hline Blood-Brain Barrier & $\mathrm{BBB}+$ & $\mathrm{BBB}+$ & $\mathrm{BBB}+$ & BBB- & BBB- \\
\hline Human Intestinal Absorption & $\mathrm{HIA}+$ & $\mathrm{HIA}+$ & HIAt & $\mathrm{HIA+}$ & HIAt \\
\hline Caco-2 Permeability & $\mathrm{Caco} 2+$ & Caco2t & $\mathrm{Caco} 2+$ & Caco2- & Caco2- \\
\hline P-glycoprotein Substrate & Non-substrate & Substrate & Non-substrate & Substrate & Non-substrate \\
\hline P-glycoprotein Inhibitor I & Non-inhibitor & Inhibitor & Inhibitor & Non-inhibitor & Non-inhilbitor \\
\hline P-glycoprotein Inhibitor II & Non-inhibitor & Non-inhibitor & Inhibitor & Non-inhibitor & Inhibitor \\
\hline $\begin{array}{l}\text { Renal Organic Cation } \\
\text { Transporter }\end{array}$ & Non-inhibitor & Non-inhibitor & Non-inhibitor & Non-inhibitor & Non-inhibitor \\
\hline CYP450 3A4 Substrate & Substrate & Substrate & Non-substrate & Substrate & Non-substrate \\
\hline CYP450 1A2 Itrhibitor & Non-inhibitor & Non-inhibitor & Inhibitor & Non-inhibitor & Inhibitor \\
\hline CYP450 2C9 Inhibitor & Non-inhibitor & Inhibitor & Non-inhibitor & Non-inhibitor & Inhibitor \\
\hline CYP450 2D6 Itrhibitor & Non-inhibitor & Non-inhilbitor & Non-inhibitor & Non-inhibitor & Non-inhilbitor \\
\hline CYP450 2C19 Inhibitor & Non-inhibitor & Inhibitor & Inhibitor & Non-inhibitor & Non-inhilbitor \\
\hline CYP450 3A4 Itrhibitor & Non-inhibitor & Inhibitor & Non-inhibitor & Non-inhibitor & Inhibitor \\
\hline $\begin{array}{l}\text { Human Ether-a-go-go- } \\
\text { Related Gene Inhibition I }\end{array}$ & Weak inhibitor & Weak inhibitor & Weak inhibitor & Weak imhibitor & Weak inhibitor \\
\hline $\begin{array}{l}\text { Human Ether-a-go-go- } \\
\text { Related Gene Inhibition II }\end{array}$ & Non-inhibitor & Non-inhibitor & Non-inhibitor & Inhibitor & Non-inhibitor \\
\hline AMES Toxicity & Non AMES toxic & Non AMES toxic & Non AMES toxic & Non AMES toxic & AMES toxic \\
\hline Carcinogens & Non-carcinogens & Non-carcinogens & Non-carcinogens & Non-carcinogens & Non-carcinogens \\
\hline Fish Toxicity & High FHMT & Low FHMT & High FHMT & High FHMT & High FHMT \\
\hline $\begin{array}{l}\text { Tetrahymena Pyriformis } \\
\text { Toxicity }\end{array}$ & High TPT & High TPT & High TPT & High TPT & High TPT \\
\hline Honey Bee Toxicity & Low HBT & Low HBT & Low HBT & Low HBT & Low HBT \\
\hline Biodegradation & $\begin{array}{c}\text { Ready } \\
\text { biodegradable }\end{array}$ & $\begin{array}{c}\text { Not ready } \\
\text { biodegradable }\end{array}$ & $\begin{array}{c}\text { Not ready } \\
\text { biodegradable }\end{array}$ & $\begin{array}{c}\text { Not ready } \\
\text { biodegradable }\end{array}$ & $\begin{array}{c}\text { Not ready } \\
\text { biodegradable }\end{array}$ \\
\hline Acute Oral Toxicity & III & III & III & III & III \\
\hline $\begin{array}{l}\text { Carcinogenicity (Three- } \\
\text { class) }\end{array}$ & Non-required & Non-required & Non-required & Non-required & Non-required \\
\hline $\begin{array}{l}\text { Rat Acute Toxicity (LD50, } \\
\text { mol/kg) }\end{array}$ & 2.3748 & 2.6009 & 2.3588 & 2.6221 & 2.1532 \\
\hline Aqueous solubility (LogS) & -2.5609 & -3.4395 & -3.6963 & -3.2513 & -3.8402 \\
\hline
\end{tabular}

Green and red entries indicate the favorable and unfavorable effects or properties of the compounds, respectively.

16814409, respectively. The 3D interaction diagram of hit compounds in the colchicine site of tubulin is comprehensively shown in Fig. 8.

\subsection{Binding free energy and leadlikeness analysis}

In order to quantify the binding affinity of hit compounds to tubulin, the binding free energies of hits were predicted by MMPBSA method. As shown in Table 2, the binding free energy of colchicine (reference ligand) was observed to be $-116.1 \mathrm{~kJ} / \mathrm{mol}$, which indicates that the present hit compounds have strong binding affinity with tubulin. Compound 17594471 interacted weakly with important residues in the active site and the binding free energy of this ligand was not significant. The binding free energies of 57367244 and 16814409 were nearly similar to the reference ligand but it was encouraging to find that compound 69899719 had higher binding free energies as compared to the reference ligand.

Table 2 In total, more than 20 highly predictive qualitative classification models were applied for the calculation of the physicochemical property of hit compounds. The ADMET properties of four of the hit compounds are shown in Table 3. The excellent ADMET properties of these hits make them promising candidates as anticancer therapeutic agents. Colchicine (as reference ligand) is a FDA-approved drug to treat gout in adults, and to treat a genetic condition called Familial Mediterranean Fever in adults and children above 4 years (Cerquaglia et al., 2005).

\section{Conclusion}

In the history of cancer treatment, tubulin inhibitors have proven to be a magnificently effective strategy to eliminate cancer cells. So, it seems necessary to identify new drugs for improving these cancer treatment methods. In this study, we used a combination of ADMET and structure-based screening approaches to identify new chromene-based inhibitors for the colchicine binding site of tubulin. At first, as the first level of filtering, RO5 and ADMET properties were applied, and then we used docking studies as second screening approach. After identifying the hit compounds, MD simulations were performed to further validate our results. MD trajectories analyses such as the RMSD, RMSF, total 
energy, $\mathrm{Rg}$ and binding free energy proved that our hit compounds (PubChem CIDs: 16814409, 17594471, 57367244 and 69899719) can prove to be potent inhibitors of tubulin. These predicted inhibitors could bind to the colchicine binding site and, lead to the depolymerization of microtubules. Reducing the growth rate of microtubules could result in catastrophic events and finally cause cell cycle arrest. In the recent years, computational drug discovery has turned into one of the most promising methods in drug discovery. This study demonstrated that how effective these methods are and how efficiently they can predict potent inhibitors for a certain molecular target.

\section{Acknowledgment}

This investigation was supported by a grant from the Ministry of Science, Research and Technology of Iran.

\section{References}

Aryapour, H., Riazi, G., Foroumadi, A., Ahmadian, S., Shafiee, A., Karima, O., Mahdavi, M., Emami, S., Sorkhi, M., Khodadady, S., 2011. Med. Chem. Res. 20, 503-510.

Aryapour, H., Riazi, G.H., Ahmadian, S., Foroumadi, A., Mahdavi, M., Emami, S., 2012a. Pharm. Biol. 50, 1551-1560.

Aryapour, H., Mahdavi, M., Mohebbi, S.R., Zali, M.R., Foroumadi, A., 2012b. Arch. Pharm. Res. 35, 1573-1582.

Berman, H.M., Westbrook, J., Feng, Z., Gilliland, G., Bhat, T.N., Weissig, H., Shindyalov, I.N., Bourne, P.E., 2000. Nucleic Acids Res. 28, 235-242.

Bietz, S., Urbaczek, S., Schulz, B., Rarey, M., 2014. J. Cheminform. 6, 12.

Bray, F., Ren, J.S., Masuyer, E., Ferlay, J., 2013. Int. J. Cancer 132, 1133-1145.

Brooks, B.R., Brooks 3rd, C.L., Mackerell Jr., A.D., Nilsson, L., Petrella, R.J., Roux, B. Won, Y., Archontis, G., Bartels, C., Boresch, S., Caflisch, A., Caves, L., Cui, Q. Dinner, A.R., Feig, M., Fischer, S., Gao, J., Hodoscek, M., Im, W., Kuczera, K., Lazaridis, T., Ma, J., Ovchinnikov, V., Paci, E., Pastor, R.W., Post, C.B., Pu, J.Z., Schaefer, M., Tidor, B., Venable, R.M., Woodcock, H.L., Wu, X., Yang, W., York, D. M., Karplus, M., 2009. J. Comput. Chem. 30, 1545-1614.

Cerquaglia, C., Diaco, M., Nucera, G., La Regina, M., Montalto, M., Manna, R., 2005 Curr. Drug Targets Inflamm. Allergy 4, 117-124.

Cheng, F., Li, W., Zhou, Y., Shen, J., Wu, Z., Liu, G., Lee, P.W., Tang, Y., 2012. J. Chem. Inf Model. 52, 3099-3105.

Curini, M., Cravotto, G., Epifano, F., Giannone, G., 2006. Curr. Med. Chem. 13, 199222.

Durrant, J.D., McCammon, J.A., 2011. BMC Biol. 9, 71.
Ertl, P., Rohde, B., Selzer, P., 2000. J. Med. Chem. 43, 3714-3717.

Essmann, U., Perera, L., Berkowitz, M.L., Darden, T., Lee, H., Pedersen, L.G., 1995. J. Chem. Phys. 103, 8577-8593.

Fiser, A., Do, R.K., Sali, A., 2000. Protein Sci. 9, 1753-1773.

W., Foye , Padova Piccin, 416 (1991).

Green, G., Evans, J., Vong, A., Katritzky, A., Rees, C., Scriven, E., 1995. Comprehensive Heterocyclic Chemistry II, vol. 5. , pp. 469.

Hess, B., Bekker, H., Berendsen, H.J.C., Fraaije, J.G.E.M., 1997. J. Comput. Chem. 18, 1463-1472.

Irwin, J.J., Shoichet, B.K., 2005. J. Chem. Inf. Model. 45, 177-182.

Khafagy, M.M., El-Wahab, A.H.A., Eid, F.A., El-Agrody, A.M., 2002. Il Farmaco 57, 715722.

Kim, S., Thiessen, P.A., Bolton, E.E., Chen, J., Fu, G., Gindulyte, A., Han, L., He, J., He, S., Shoemaker, B.A., Wang, J., Yu, B., Zhang, J., Bryant, S.H., 2016. Nucleic Acids Res. 44, D1202-1213.

Kumari, R., Kumar, R., Open Source Drug Discovery Consortium, Lynn, A., 2014. J. Chem. Inf. Model. 54, 1951-1962.

Lipinski, C.A., Lombardo, F., Dominy, B.W., Feeney, P.J., 2001. Adv. Drug Deliv. Rev. 46, 3-26.

M.I.O.S, 2006. Drug Discov. Today 3, 405-411.

Muegge, I., 2008. Mini. Rev. Med. Chem. 8, 927-933.

Mungra, D.C., Patel, M.P., Rajani, D.P., Patel, R.G., 2011. Eur. J. Med. Chem. 46, 41924200.

Patil, S.A., Patil, R., Pfeffer, L.M., Miller, D.D., 2013. Future Med. Chem. 5, 1647-1660.

Prota, A.E., Danel, F., Bachmann, F., Bargsten, K., Buey, R.M., Pohlmann, J., Reinelt, S., Lane, H., Steinmetz, M.O., 2014. J. Mol. Biol. 426, 1848-1860.

Saffari, Z., Aryapour, H., Akbarzadeh, A., Foroumadi, A., Jafari, N., Farahnak Zarabi, M., Farhangi, A., 2014. Tumour Biol. J. Int. Soc. Oncodev. Biol. Med. 35, 5845-5855.

Shao, C.Y., Su, B.H., Tu, Y.S., Lin, C., Lin, O.A., Tseng, Y.J., 2015. Bioinformatics 31,18691871.

Smith, P.W., Sollis, S.L., Howes, P.D., Cherry, P.C., Starkey, I.D., Cobley, K.N., Weston, H., Scicinski, J., Merritt, A., Whittington, A., 1998. J. Med. Chem. 41, 787-797. Stierand, K., Rarey, M., 2007. Chem. Med. Chem. 2, 853-860.

Tanrikulu, Y., Kruger, B., Proschak, E., 2013. Drug Discov. Today 18, 358-364.

Temraz, S., Mukherji, D., Alameddine, R., Shamseddine, A., 2014. Crit. Rev. Oncol. Hematol. 89, 217-230.

Trott, O., Olson, A.J., 2010. J. Comput. Chem. 31, 455-461.

Van Der Spoel, D., Lindahl, E., Hess, B., Groenhof, G., Mark, A.E., Berendsen, H.J., 2005. J. Comput. Chem. 26, 1701-1718.

Veber, D.F., Johnson, S.R., Cheng, H.-Y., Smith, B.R., Ward, K.W., Kopple, K.D., 2002. J. Med. Chem. 45, 2615-2623.

Walters, W.P., Stahl, M.T., Murcko, M.A., 1998. Drug Discov. Today 3, 160-178.

Wang, R., Lai, L., Wang, S., 2002. J. Comput. Aided Mol. Des. 16, 11-26.

Wang, T., Wade, R.C., 2003. Proteins Struct. Funct. Bioinformatics 50, 158-169.

Waxman, D.J., Schwartz, P.S., 2003. Cancer Res. 63, 8563-8572.

Zoete, V., Cuendet, M.A., Grosdidier, A., Michielin, O., 2011. J. Comput. Chem. 32 2359-2368. 